\title{
Desarrollo y validación de un método ambientalmente amigable para determinación de metales pesados en pastos
}

\author{
Development and validation of environmental friendly method for determination \\ of heavy metals in pastures
}

Amanda Lucia Chaparro G. ${ }^{1}$; Jhon Jairo García F. ${ }^{2}$; Yaneth Cardona R. ${ }^{3}$; Jhon Jairo Bustamante C. ; Manuel Peláez P.

1 Docente. Ph.D. Universidad de Pamplona, Pamplona, Colombia, achaparro@unipamplona.edu.co.

${ }^{2}$ Químico. Universidad de Pamplona, Pamplona, Norte de Santander, Colombia, jhongarciaff@gmail.com.

${ }_{3}^{3}$ Docente. M.Sc. Universidad de Pamplona, Pamplona, Colombia, yaneth.cardona@unipamplona.edu.co.

${ }^{4}$ Docente. Ph.D. Universidad de Pamplona, Pamplona, Colombia, jhonjabu@unipamplona.edu.co.

${ }^{5}$ Docente.Ph.D.Universidad Nacional de Colombia, Sede Palmira, Palmira, Colombia, jpelaezp@unal.edu.co.

Citar: CHAPARRO, AL.; GARCIA, JJ.; CARDONA, Y.; BUSTAMANTE, JJ.; PELAEZ, MJ. Desarrollo y validación de un método ambientalmente amigable para determinación de metales pesados en pastos. Rev. Cienc. Agr. 33(2):3-15. doi:http://dx.doi.org/10.22267/rcia.163302.48.

Recibido: Agosto $082015 \quad$ Aceptado: Mayo 20 de 2016

\section{RESUMEN}

Los metales pesados son peligrosos para los seres vivos y el medio ambiente, pues no pueden ser degradados y pueden llegar a los seres humanos a través la cadena trófica, por lo tanto, su determinación en diferentes matrices para consumo humano y animal, es de vital importancia. La metodología más utilizada para su extracción, es cenizas, la cual conlleva a tiempos largos de análisis y elevado consumo de energía, por esta razón, en el presente trabajo se desarrolló y validó un método ambientalmente amigable para la digestión y extracción de $\mathrm{Cd}^{+2} \mathrm{y} \mathrm{Pb}^{+2}$ en pastos, utilizando ultrasonido y espectroscopia de absorción atómica. Se evaluaron diferentes variables que afectan la digestión, como posición en el ultrasonido, peso de muestra, solvente, volumen del solvente, tiempo de sonicación y tipo de recipiente, en muestras de pastos dopadas con los metales, teniéndose como variable de respuesta, el porcentaje de recuperación. Las condiciones para extraer $\mathrm{Cd}^{+2} \mathrm{y} \mathrm{Pb}^{+2}$ en estas pasturas, fueron: $0,50 \mathrm{~g}$ de tejido vegetal; 3,00 mL de $\mathrm{HCl}: \mathrm{HNO}_{3}-3: 1$ expuestos $60 \mathrm{~min}$ al ultrasonido, en tubos de vidrio cónicos. Este procedimiento mostró un comportamiento lineal en el rango de 0,50 - 8,00mg/Kg con $\mathrm{R}^{2}$ de 0,99 tanto para Cd como para $\mathrm{Pb}$. Los límites de detección y cuantificación, fueron 0,25 y 0,43mg/Kg para Cd; 0,22 y 0,37mg/Kg para $\mathrm{Pb}$. La metodología propuesta, se comparó con la técnica clásica y permitió determinar niveles trazas de estos metales tóxicos, en forraje proveniente del Magdalena medio, con porcentajes de recuperación de 97,54 y $98,62 \%$, respectivamente.

Palabras clave: Cadmio, digestión, extracción, plomo, ultrasonido. 


\begin{abstract}
Heavy metals are dangerous for living beings and environment, because they cannot be degraded or destroyed. They can reach human beings through the food chain; therefore, their determination in different matrices for human and animal consumption is very important. The method currently used for their extraction is by ash, which entails long processing times and high energy consumption. For this reason, in this study, an environmental friendly method, for digestion and extraction $\mathrm{Cd}^{+2}$ and $\mathrm{Pb}^{+2}$, in pastures, was developed and validated; using ultrasound and atomic absorption spectroscopy. Different variables that affect the digestion were evaluated, such as position on the ultrasound, sample weight, solvent, solvent volume, sonication time and type of container, in samples of pastures doped with metals, using recovery rate as a response variable. The conditions for digestion and extraction of $\mathrm{Cd}^{+2}$ an $\mathrm{Pb}^{+2}$ were: $0.50 \mathrm{~g}$ of grass in $3.00 \mathrm{~mL}$ of aqua regia $\left(\mathrm{HCl}_{\mathrm{HNO}}-3: 1\right)$ exposed for $60 \mathrm{~min}$ to ultrasound in conical glass tubes. The proposed procedure showed a linear behavior in the range of 0.50 to $8.00 \mathrm{mg} / \mathrm{Kg}$, with 0.9968 and 0.9999 of $\mathrm{R}^{2}$ for $\mathrm{Cd}^{+2}$ and $\mathrm{P}^{+2} \mathrm{~b}$ respectively. The limits of detection and quantification were $0.25-0.43 \mathrm{mg} / \mathrm{Kg}$ for $\mathrm{Cd}^{+2}$ and $0.22-0.37 \mathrm{mg} / \mathrm{Kg}$ for $\mathrm{Pb}^{+2}$. The proposed methodology was compared with the conventional technique, and this method allowed determining trace levels of these toxic metals in a forage sample from Magdalena region, with recoveries of $97.54 \%$ and $98.62 \%$, respectively.
\end{abstract}

Keywords: Cadmium, digestion, extraction, lead, ultrasound.

\section{INTRODUCCION}

El estilo de vida de la sociedad actual ha conllevado al impulso de las diversas actividades antropogénicas e industriales y por ende, a un aumento en la emisión de sustancias contaminantes, generando la acumulación de éstas en suelos, agua y plantas, que están íntimamente relacionados con las especies animales; lo cual, facilita la entrada de dichas sustancias tóxicas a las redes tróficas. Dentro de los contaminantes ambientales se encuentran los metales pesados, término que suele asignarse a elementos con número atómico por encima de 20 y peso específico superior a $5 \mathrm{~g} / \mathrm{cm}^{3}$. Algunos de estos metales son benéficos, debido al papel que desempeñan en las funciones bioquímicas y fisiológicas en los seres vivos y otros pueden ser muy tóxicos o inclusive letales en concentraciones muy bajas (Cobb et al., 2000; Negroni, 2009; Rascio y Navari, 2011; Tchounwou et al., 2012; Wang et al., 2015; Bonanno et al., 2017).
La propagación de los metales pesados en la cadena alimenticia, constituye un riesgo serio para el medio ambiente y la salud de plantas, animales y humanos (Minkina et al., 2016; Tóth et al., 2016), puesto que son sustancias con una gran estabilidad química ante los procesos de biodegradación y no son metabolizados por los seres humanos, generan bioacumulación y biomagnificación de su concentración en la red trófica (Navarro et al., 2007; Kabata, 2011; Mollon et al., 2016; Tóth et al., 2016). Dentro de los metales que representan serios problemas medioambientales, se encuentran el mercurio (Hg), molibdeno $(\mathrm{Mo})$, plomo $(\mathrm{Pb})$, cadmio $(\mathrm{Cd})$, talio $(\mathrm{Tl})$, vanadio (V) y algunos metaloides como selenio (Se) y arsénico (As) por esta razón, en los últimos años se ha despertado el interés de su estudio y regulación (Ferré et al., 2007; Prieto et al., 2009; Minkina et al., 2016).

La concentración de estos metales pesados en la naturaleza, se aumenta con la aplicación de biosólidos, fertilizantes y la irrigación con aguas 
contaminadas, las cuales, alteran suelos agrícolas, aguas subterráneas y agroecosistemas como las pasturas que bioconcentran dichos metales tóxicos, llegando de ésta manera a causar patologías en los tejidos animales una vez se magnifican. Posteriormente, estos tóxicos se transfieren a la dieta del hombre por consumo directo de vegetales como frutas y hortalizas o indirecto con la ingesta de productos cárnicos, trayendo repercusiones en la salud pública (Miranda et al., 2008; Duong y Byeong, 2011).

Para la determinación de estos contaminantes ambientales en diferentes muestras de tejido vegetal, se emplean protocolos tradicionales, como, la digestión ácida por vía húmeda y seca. La vía húmeda, consiste en utilizar ácidos, bases y agentes oxidantes, para asegurar la total destrucción de la materia orgánica y la vía seca, se fundamenta en procesos de calcinación en muflas, seguida de tratamiento con estas mismas sustancias. No obstante, estas técnicas requieren de un tiempo considerable de análisis y volúmenes elevados de ácidos, lo que contribuye a impactar negativamente al medio ambiente con emisiones de vapores y residuos tóxicos.

Por otra parte, se pueden presentar problemas de disolución incompleta, precipitación de analitos insolubles, contaminación de la muestra y perdida de elementos volátiles (Kazi et al., 2009; Merey et al., 2002). Debido a estos inconvenientes, se han propuesto otros procedimientos para la preparación de muestras vegetales, como digestión asistida por microondas y ultrasonido, sin embargo, el equipo de la digestión asistida por microondas es costoso y limita su aplicación (Bakkali et al., 2009; Soylak et al., 2007). Por otra parte, la energía empleada en la digestión asistida por ultrasonido, facilita y acelera algunos pasos, tales como la disolución, fusión y lixiviación, entre otros; por lo cual, ha sido utilizada para la preparación de muestras en aplicaciones agríco- las, biológicas y ambientales (Priego y Luque de Castro, 2007a).

Por lo anterior, el propósito de ésta investigación, fue desarrollar y validar un método amigable con el medio ambiente, para la extracción de $\mathrm{Cd}$ y $\mathrm{Pb}$ en forrajes, realizando la cuantificación de los mismos, por espectroscopia de absorción atómica de llama.

\section{MATERIALES Y MÉTODOS}

Para el presente estudio se utilizaron estándares analíticos de Cd (Merck $\AA$ ) y Pb (Carlo Erba $囚$ ) a una concentración de 1000 mg/L. A partir de estos, se prepararon las soluciones de dopaje $(200 \mathrm{mg} / \mathrm{L})$ de cada metal y soluciones patrón de $0,50 \mathrm{mg} / \mathrm{L}$ a 10,00mg/L de Cd y Pb para las curvas de calibración. Adicionalmente, para las digestiones se utilizó ácido clorhídrico fumante al 37\% (Merck®), ácido nítrico al 65\% (Merck®) y una mezcla de estos $\mathrm{HCl}: \mathrm{HNO}_{3}$ en proporción 3:1.

Los procesos de digestión y extracción se llevaron a cabo en un baño de ultrasonido BRANSON 1510R$M T$ con una potencia de 70W. La determinación y cuantificación de los metales se realizó con un espectrómetro de absorción atómica con atomización en llama Perkin Elmer AAnalyst 300, utilizando lámparas de cátodo hueco de $\mathrm{Cd}$ y $\mathrm{Pb}$ como fuente de energía y una llama oxidante compuesta por aire-acetileno (10:1). Todos los ensayos se llevaron a cabo por triplicado.

Desarrollo del método. Para la elección de las condiciones favorables para la extracción de $\mathrm{Cd}$ y $\mathrm{Pb}$, el material vegetal utilizado (Pennisetum clandestinum Hochst. ex Chiov) libre de contaminación, se dopó adicionando $1 \mathrm{~mL}$ (200mg/L de cada metal) y se dejó en reposo durante 24 horas antes de su análisis. Se evaluaron diferentes parámetros que afectan el proceso de extracción tales como: posición en el baño de 
ultrasonido, solvente y mezcla de solventes $(\mathrm{HCl}$, $\mathrm{HNO}_{3}$ y agua regia), peso de la muestra $(0,50$; $1,00 ; 1,50$ y $2,00 \mathrm{~g})$, volumen del solvente $(1,00$; 3,00 y $5,00 \mathrm{ml}$ ), tiempo de ultrasonido (periodos de $10 \mathrm{~min}$ hasta completar $60 \mathrm{~min}$, posteriormente se realizó un análisis en periodos de tiempo de $1 \mathrm{~h}$ hasta completar $4 \mathrm{~h}$ ) y tipo de recipiente (polipropileno y vidrio, entre los primeros se encuentran tubos de centrifuga de $15 \mathrm{~mL}$ cónicos y vasos fondo plano de $25 \mathrm{~mL}$, y entre los de vidrio están beakers de $50 \mathrm{~mL}$, tubo de centrifuga $15 \mathrm{~mL}$ cónico y tubo tapa rosca punta redonda).

Finalmente, el extracto se filtró y se aforó a $50 \mathrm{~mL}$ con agua destilada. Para observar de manera uniforme el comportamiento de los metales en cada una de las pruebas, se realizó la normalización de los resultados, con base al máximo valor de recuperación del metal en cada variable evaluada.

El método desarrollado, se comparó con el método tradicional de digestión vía seca, realizando el análisis en una muestra expuesta a la contaminación atmosférica (con y sin lavar la misma antes del análisis), tomada en los alrededores de zonas de explotación petrolera de Barrancabermeja, en el Magdalena medio colombiano. Para el método tradicional se siguió la metodología descrita en el manual "Analytical Methods for Atomic Absorption Spectroscopy" del equipo de absorción atómica Perkin Elmer AAnalyst 300: 1,00g de pasto se llevó a $500^{\circ} \mathrm{C}$ en un horno durante 16 horas, luego se adicionaron 5,00mL de ácido clorhídrico (37\%) y se llevó a calentamiento hasta disolver las cenizas, finalmente se filtró y aforó a 50,00mL con agua destilada.

Análisis estadístico. Se realizó el Análisis de Varianza (ANDEVA) $(\alpha=0,05)$ en la elección de cada condición, empleando el paquete estadístico OriginPro 8,6 (versión de prueba), con la finalidad de encontrar la existencia de diferencias estadísticamente significativas y conocer entre que grupos se dan dichas diferencias.

\section{RESULTADOS Y DISCUSIÓN}

El método asistido por ultrasonido para la extracción de $\mathrm{Pb}$ y $\mathrm{Cd}$ en pastos que se presenta en este trabajo, es más rápido (1 hora) que las técnicas tradicionales (16-24 horas) y consume solamente $3 \mathrm{~mL}$ de solvente; lo cual, equivale al 50\% - 99\% de las cantidades empleadas en las técnicas tradicionales, esto, a su vez reduce la generación de desechos en la misma proporción. La metodología planteada, reduce adicionalmente el consumo de energía, pues disminuye el tiempo de uso de los equipos eléctricos: 60 minutos de ultrasonido conectado a $110 \mathrm{~V}$, versus 16 horas de una mufla conectada a $220 \mathrm{~V}$ en digestión vía seca, haciéndolo un método amigable con el medio ambiente y la salud.

\section{Condiciones de digestión y extracción}

Posición en el baño de ultrasonido. Dentro de las diferentes variables que afectan la digestión de la muestra por ultrasonido, se encuentra la posición de las muestras dentro del equipo, lo cual puede variar la uniformidad de la energía que emite el transductor (Kanthale et al., 2003; Priego y Luque de Castro, 2007a); sin embargo, la recuperación de los metales $\mathrm{Cd}$ y $\mathrm{Pb}$ del pasto dopado en éste estudio, no presentó diferencias estadísticamente significativas $(\mathrm{p}>0,05)$ en las diferentes ubicaciones evaluadas (Figura 1), por lo cual, la posición en el baño de ultrasonido marca Branson 1510 de 2L no influye significativamente en la recuperación de los mismos y por ello, se procedió a utilizar este equipo para la digestión y extracción en cualquier posición.

Selección del ácido o mezcla de ácidos. Los solventes tienen propiedades particulares que afectan la digestión asistida por ultrasonido, algunos se oponen al umbral de cavitación (Priego y Luque de Castro, 2007a). 


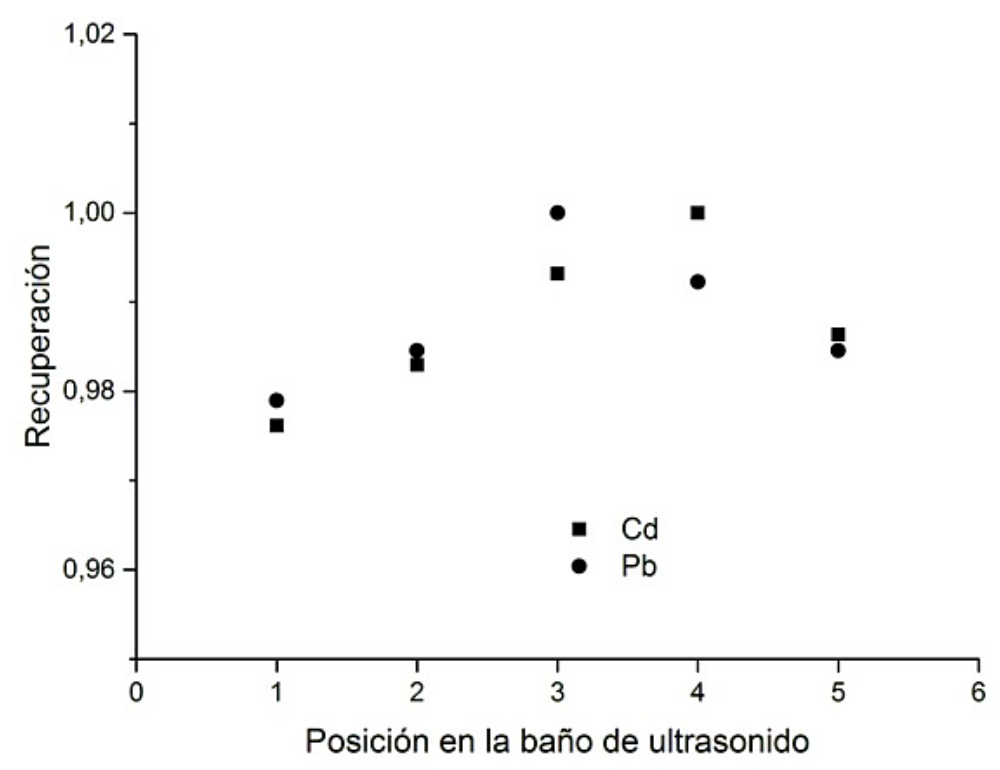

Figura 1. Efecto de la posición en el baño de ultrasonido en la recuperación

De otra forma, es importante tener en cuenta, que el comportamiento del solvente no es el mismo con respecto a cualquier matriz y analito, por lo tanto, algún fenómeno que modifique las propiedades del solvente como viscosidad, solubilidad o temperatura critica, pueden modificar este umbral (Elik, 2005; Priego y Luque de Castro, 2007a). Los valores normalizados de recuperación para cada uno de los metales con respecto al tipo de ácido utilizado, se encuentran en la Figura 2. Como puede observarse, utilizando agua regia $\left(\mathrm{HCl}: \mathrm{HNO}_{3}-3: 1\right)$, se obtuvo una mejor recuperación para $\mathrm{Cd}$ y $\mathrm{Pb}$, por lo tanto, se procedió a trabajar con esta mezcla de ácidos para los demás ensayos realizados.

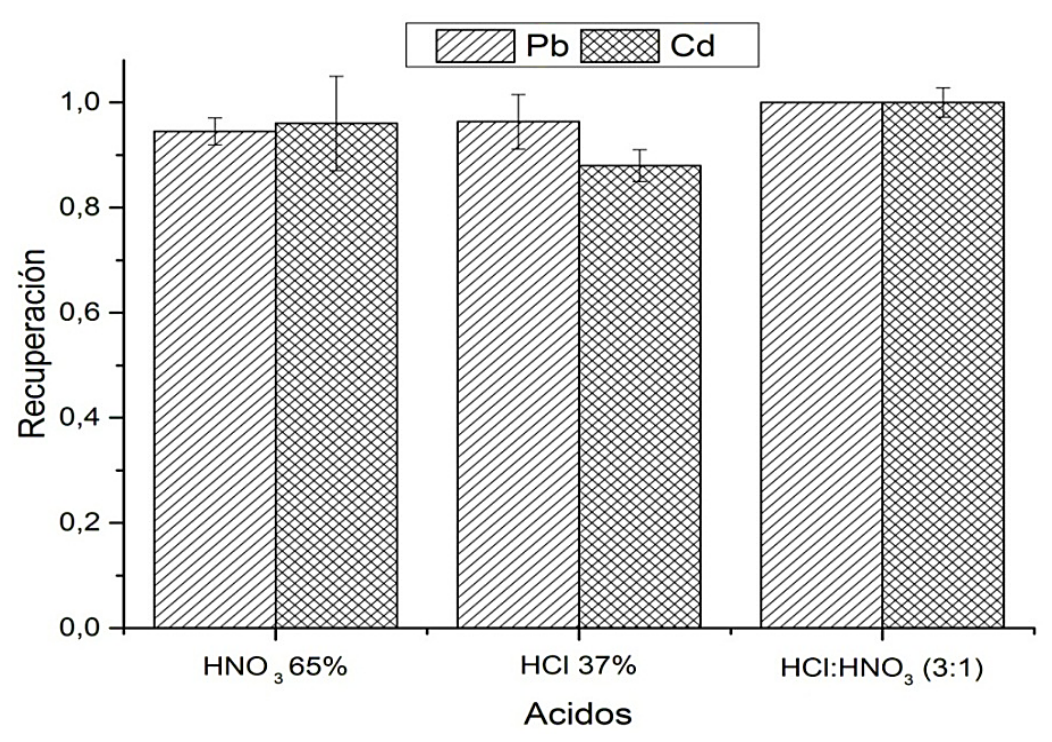

Figura 2. Recuperación de los metales evaluados, debido a los diferentes ácidos empleados en el proceso de extracción. 
Peso de la muestra. En la Figura 3, se puede observar que existe mejor recuperación de $\mathrm{Cd}$ y $\mathrm{Pb}$ utilizando 0,50 g de pasto. Esta es una cantidad menor a la utilizada en los métodos tradicionales, que generalmente requieren entre uno a dos gramos. En este ensayo, se pudo observar que la canti- dad de muestra influye en la recuperación de estos metales; lo cual, se debe a que los efectos de las ondas de ultrasonido se concentran en esta menor cantidad de muestra, acelerando procesos de disolución, fusión y lixiviación, permitiendo de esta forma, una mayor recuperación del analito.

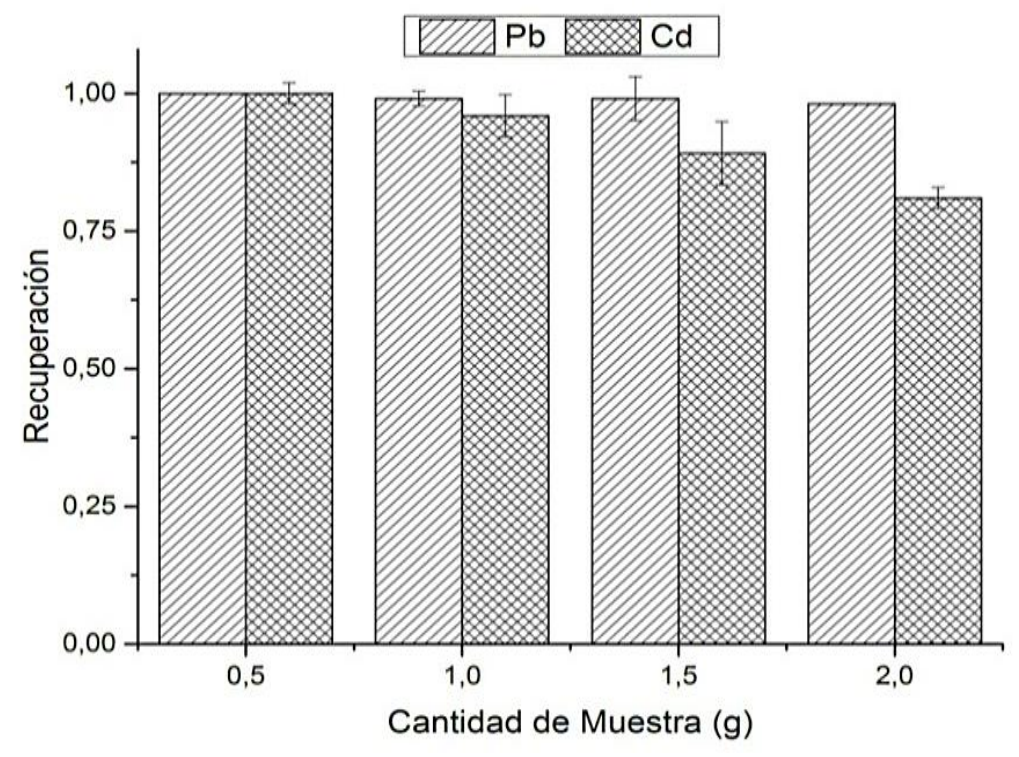

Figura 3. Recuperación de los metales evaluados, empleando diferentes cantidades de muestra en el proceso de extracción.

Volumen de ácido. En trabajos realizados por Brunori et al. (2004) y Nascentes et al. (2001), se evidenció, que el volumen de los ácidos y la cantidad de muestra influyen en los límites de detección, puesto que la relación masa/volumen de solvente varía, quedando en algunos casos una gran cantidad de solvente en poca muestra 0 viceversa, adicionalmente esto se ha confirmado por Priego y Luque de Castro (2007a), donde evalúan los diferentes parámetros que pueden afectar este proceso.
En esta investigación, se encontró que para el Cd y $\mathrm{Pb}$ las diferencias no son estadísticamente significativas ( $p>0,05)$ entre los diferentes volúmenes evaluados (Figura 4); sin embargo, al utilizar 1,0mL se favorecía la recuperación del $\mathrm{Pb}$ y al utilizar $5,0 \mathrm{~mL}$ la del Cd, por lo cual se decidió trabajar con un volumen de 3,0mL. Adicionalmente, se tuvo en cuenta el hecho que al disminuir la cantidad de muestra empleada $(0,5 \mathrm{~g})$, un volumen de $5,0 \mathrm{~mL}$, resultaba excesivo en el proceso de extracción. 


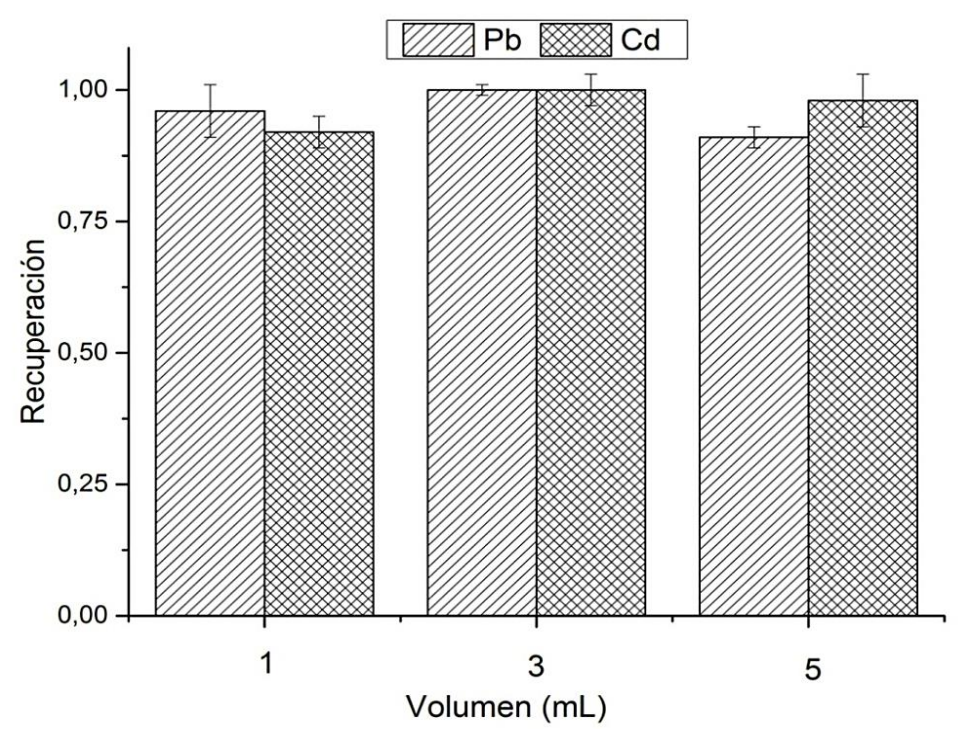

Figura 4. Recuperación de los metales evaluados, debido a diferentes volúmenes de agua regia.

Efecto del tiempo de sonicación. En la Figura 5 , se puede observar que el tiempo óptimo para la extracción de los metales estudiados fue de $60 \mathrm{~min}$, pues a partir de ese tiempo, disminuye la recuperación de los mismos. De acuerdo con algunos autores, la optimización del tiempo de sonicación es muy importante, ya que un tiempo más prolongado, podría dar lugar a la reducción de los elementos o formación de radicales libres, que a su vez son muy reactivos y formarían parte de una serie de reacciones que involucran a los metales pesados, interfiriendo en el análisis (Brunori et al., 2004). Esto podría explicar, la disminución de la recuperación después de una hora de expuesto el material al ultrasonido en ésta investigación. Por otra parte, la disminución del tiempo reduce los costos, debido a que el analista puede realizar mayor cantidad de ensayos en menor tiempo, con menos consumo eléctrico y desgaste de los equipos de trabajo.

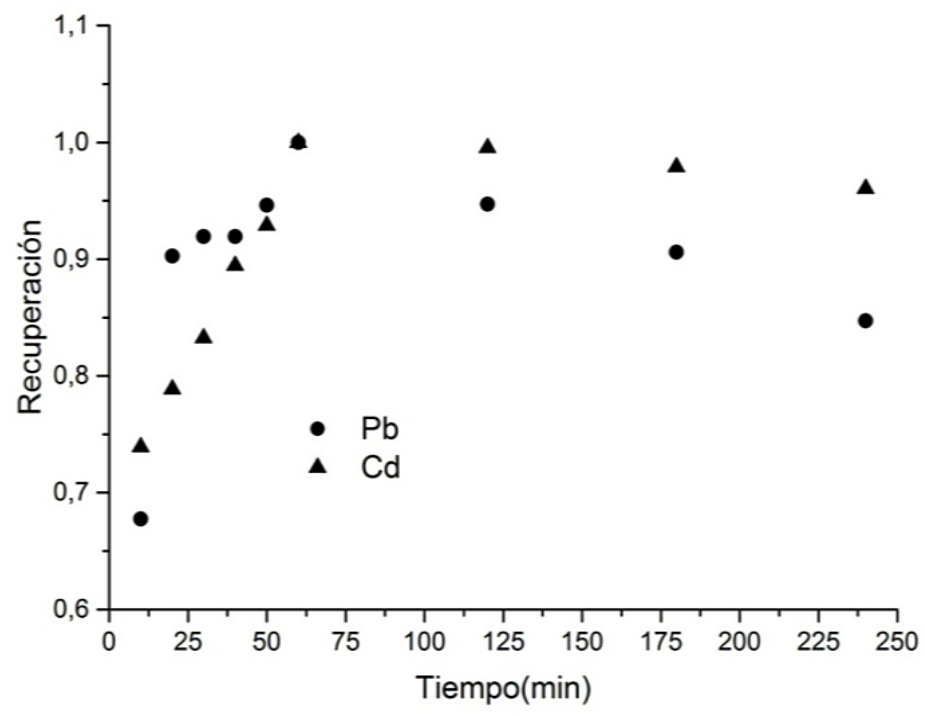

Figura 5. Recuperación de los metales evaluados a diferentes tiempos de sonicación. 
Tipo de recipiente. El hecho que un metal no sea afectado por las ondas de ultrasonido y sus efectos, puede deberse al material, en el cual se realizan los procesos de digestión, puesto que el grosor, la composición y la forma de estos, obstaculizan las ondas ultrasónicas y a su vez, disminuyen el efecto que realiza en la muestra, siendo menos efectiva la transferencia de energía (Priego y Luque de Castro, 2007b; Cespón y Yebra, 2008). En éste trabajo, se encontró una mejor recuperación de metales con tubos de vidrio punta fina (Figura 6), lo cual favorece al método en sí, pues se puede analizar simultáneamente, un mayor número de muestras en el ultrasonido.

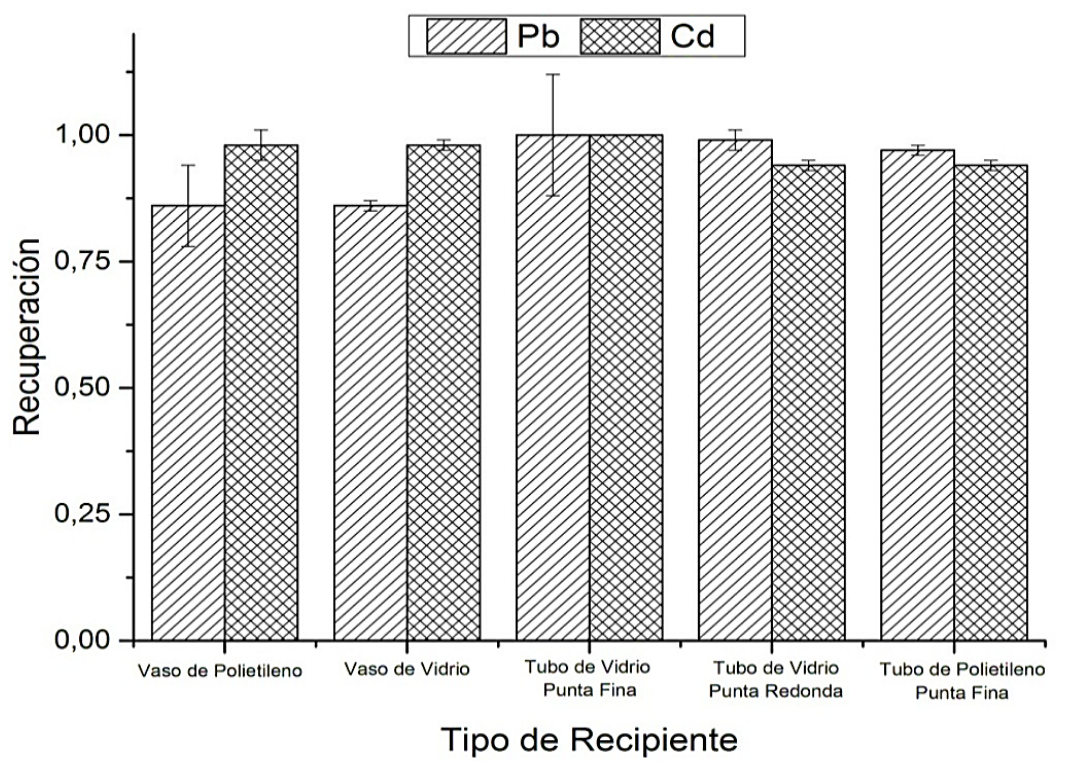

Figura 6. Recuperación de los metales evaluados al emplear diferentes recipientes en el proceso de extracción.

Al comparar los resultados de esta investigación con metodologías tradicionales, se pueden observar que las condiciones encontradas, son una alternativa favorable para el análisis de estas muestras, puesto que reducen el volumen de ácido empleado, el tiempo, el consumo de energía y la contaminación que se genera en éste tipo de procesos.

Análisis estadístico. Mediante el ANDEVA simple, se encontró que no hay diferencias estadísticamente significativas entre los análisis realizados dentro de una misma prueba $(p>0,05)$, pero sí hay diferencias entre las variables comparadas $(\mathrm{p}<0,05)$ en cada ensayo, con un nivel del 95\% de confianza, a excepción de las pruebas realizadas en las variables, posición del baño de ultrasonido y volumen de ácido empleado, en las cuales no se encontraron diferencias estadísticamente significativas $(p>0,05)$ entre las valores evaluados.

Validación del método propuesto. Una vez evaluados las diferentes variables que afectan la digestión por ultrasonido se encontró, que las mejores condiciones para la extracción de $\mathrm{Cd}$ y $\mathrm{Pb}$ en pastos fueron: $0,50 \mathrm{~g}$ de pasto en $3,00 \mathrm{~mL}$ de $\mathrm{HCl}: \mathrm{HNO}_{3}$ (3:1), expuestos durante $60 \mathrm{~min}$ al ultrasonido en tubos de vidrio cónicos, seguidos de un aforo a $50,00 \mathrm{~mL}$ con agua destilada. Con estas condiciones ajustadas, se procedió a realizar la validación de este método para análisis de $\mathrm{Cd}$ y $\mathrm{Pb}$ en muestras de pasto $P$. clandestinum, dopadas con solución estándar de cada uno de los metales. Los parámetros de validación son presentados en la Tabla 1. 
Tabla 1. Datos de validación para la digestión y extracción de $\mathrm{Cd}$ y Pb en pastos.

\begin{tabular}{lll}
\hline \multicolumn{1}{c}{ Párametro } & \multicolumn{1}{c}{ Cd } & \multicolumn{1}{c}{ Pb } \\
\hline Ecuación & $\mathrm{Y}=0,1005 \mathrm{X}+0,0076$ & $\mathrm{Y}=0,0148 \mathrm{X}-0,0022$ \\
Rango Lineal (mg/Kg) & $0,50-8,00$ & $0,50-8,00$ \\
$\mathrm{~N}$ & 6 & 6 \\
$\mathrm{~m}$ & 0,1005 & 0,0148 \\
$\mathrm{~b}$ & 0,0076 & $-0,0022$ \\
$\mathrm{R}^{2}$ & 0,9968 & 0,9999 \\
$\mathrm{LOD}(\mathrm{mg} / \mathrm{Kg})$ & 0,25 & 0,22 \\
LOQ (mg/Kg) & 0,43 & 0,37 \\
Recuperación & $97,54 \%$ & $98,62 \%$ \\
RSD & $8,15 \%$ & $7,49 \%$ \\
\hline
\end{tabular}

Linealidad, límite de detección (LOD) y límite de cuantificación (LOQ). La linealidad del método, fue evaluada utilizando 12 concentraciones diferentes en el rango $0,50-8,00 \mathrm{mg} / \mathrm{Kg}$, obteniendo un coeficiente de correlación $\left(\mathrm{R}^{2}=\right.$ coeficiente e determinación) que exhibe una buena linealidad en el rango de concentraciones examinadas (Tabla 2).

Los valores calculados de LOD (proporción señal a ruido de $3 ; \mathrm{S} / \mathrm{N}=3$ ) fueron $0,25 \mathrm{mg} / \mathrm{Kg}$ para $\mathrm{Cd}$ y $0,22 \mathrm{mg} / \mathrm{Kg}$ para Pb. Para LOQ (proporción señal a ruido de $10 ; \mathrm{S} / \mathrm{N}=10$ ) se encontraron los valores de 0,43 y $0,47 \mathrm{mg} / \mathrm{Kg}$ para $\mathrm{Cd}$ y $\mathrm{Pb}$, respectivamente.

Exactitud, precisión y repetitividad. La evaluación de la exactitud del método, se basó en la recuperación de cantidades conocidas de $\mathrm{Cd}$ y $\mathrm{Pb}$ en muestras con tres concentraciones diferentes, que fueron analizadas por triplicado de acuerdo con las condiciones favorables. La precisión del método, fue evaluada en un mismo día, analizando muestras $(\mathrm{n}=6)$ de pasto dopadas a una concentración de $4 \mathrm{mg} / \mathrm{Kg}$.

Los valores obtenidos de recuperación de metales pesados $(\mathrm{Cd}$ y $\mathrm{Pb}$ ) y desviación estándar relativa (RSD) reportados en la Tabla 2, son considerados adecuados para el análisis de metales pesados en pastos (Miller y Miller, 2000; Skoog et al., 2005), siendo satisfactoria la reproducibilidad de este método y pudiendo ser utilizado para el análisis de $\mathrm{Cd} \mathrm{y} \mathrm{Pb}$ en muestras de pastos.

Comparación del método desarrollado con el método tradicional (Cenizas). La determinación de metales a nivel de traza mediante métodos espectroscópicos, requiere de la remoción de materia orgánica, que se puede realizar mediante varios métodos tradicionales (extracción líquido-líquido y digestiones, entre otros); siendo el método más utilizado, la calcinación por vía seca en una mufla, por lo cual en este trabajo decidió compararse este método con el desarrollado. Se analizó una muestra de pasto proveniente de una zona de explotación petrolera de la región del Magdalena medio, lavada y sin lavar antes de su análisis, para determinar la presencia de los metales analizados en la superficie del tejido vegetal. Dichos análisis se llevaron a cabo por el método validado y el método tradicional por vía seca, obteniendo resultados muy similares en cuanto a la concentración de $\mathrm{Cd}$ y $\mathrm{Pb}$ en la muestra. En la Tabla 2 se presenta la comparación entre las mediciones en $\mathrm{mg} / \mathrm{Kg}$ entre los dos métodos, en cuanto al $\mathrm{Cd}$ por el método de ultrasonido existe una pequeña disminución en la recuperación, comparado con el método tradicional, por el contrario, el $\mathrm{Pb}$ presenta un aumento en la recuperación por el método validado. Adicionalmente, se puede observar una disminución en 
la concentración de metales al lavar la muestra, lo que implica que parte de la concentración de los mismos se encontraba en la superficie de la planta, procedente del medio ambiente y la otra parte, es transferida del suelo a la misma.

El método propuesto utiliza una menor cantidad de ácidos y se producen menos vapores y desechos tóxicos, lo que conlleva a que esta metodología tenga un menor impacto ambiental en comparación con la metodología tradicional. Otro punto a favor es la disminución del tiempo de pretratamiento de la muestra de 16 horas en el método tradicional, sin incluir la disolución de las cenizas, a una hora. Adicionalmente, en muflas, solamente se pueden procesar tres muestras a la vez (por duplicado); por la metodología propuesta, en un baño de ultrasonido de $2 \mathrm{~L}$, se puede tratar más de doce muestras al tiempo (por duplicado) en tan solo una hora y los resultados son similares.

Al comparar el tiempo de análisis de la técnica estandarizada y validada con los tiempos encontrados por otros autores que han utilizado ultrasonido como técnica de extracción, se encuentra variación. Es importante tener en cuenta que el tiempo necesario para la recuperación total de los metales pesados por ultrasonido, también es afectado por la matriz, el analito, el tamaño de partícula, la temperatura, la concentración de los ácidos, y el tipo de ultrasonido utilizado (sonda o baño). En este sentido, Jamali et al. (2007) observaron que en la extracción de $\mathrm{Cd}, \mathrm{Cr}, \mathrm{Ni}, \mathrm{Pb}$ y $\mathrm{Zn}$ en muestras de suelo contaminado, se requirieron de 10-20min a temperaturas de 70 a $90^{\circ} \mathrm{C}$, mientras que $\mathrm{Cu}$ requirió un tiempo de sonicación mayor $(30 \mathrm{~min})$ a $90^{\circ} \mathrm{C}$. Por otra parte Ruiz et al. (2003), encontraron que para $0,25 \mathrm{~g}$ de muestra utilizando $\mathrm{HNO}_{3}$ al $8 \%$ y utilizando un ultrasonido de sonda, pudieron extraer $\mathrm{Cd}$ y $\mathrm{Pb}$ en muestras de hojas de olivo con eficiencias de extracción para los metales en un rango del 96,5 al $103,5 \%$ para Cd y 96,88 a $98,24 \%$ para $\mathrm{Pb}$, en un tiempo de $5 \mathrm{~min}$.

Figueiras et al. (2001) utilizaron una sonda ultrasónica por $3 \mathrm{~min}$ para la extracción de metales (Ca, Cd, Mg, Mn, Pb y Zn) de tejido vegetal (0,1g, tamaño de partícula 50 micras) en una solución alcalina $(0,1 \mathrm{M}$ EDTA a $\mathrm{pH} 10)$ y posterior determinación por FAAS y ETAAS. Adicionalmente, Aleixo et al. (2004) mostraron que las mejores condiciones para la extracción ultrasónica dependían de las interacciones analito-matriz, el tipo de matriz y la técnica de cuantificación utilizada; así, ellos necesitaron concentraciones de ácido más altas para la extracción de Pb que para de Cd a partir de tejidos vegetales, mientras que ambos analitos se extrajeron con la misma concentración de ácido en los tejidos animales. Si bien el uso de sondas frente a baños de ultrasonidos reduce al mínimo la falta de homogeneidad de la distribución de energía y por ende el tiempo de sonicación, tienen la desventaja de ser más costosas, vidas más cortas y permiten llevar a cabo un número reducido de muestras, pues solo se puede analizar una muestra a la vez.

Tabla 2. Comparación entre la recuperación de metales por el método tradicional y el método validado en muestras de pastos expuestas a contaminación ambiental.

\begin{tabular}{ccccc}
\hline Método & Metal & Sin Lavar (mg/Kg) & Lavado (mg/Kg) \\
\hline Tradicional & $\mathrm{Cd}$ & 1,76 & 0,95 \\
(CENIZAS) & $\mathrm{Pb}$ & 1,44 & 0,80 \\
\hline \multirow{2}{*}{ Ultrasonido } & $\mathrm{Cd}$ & 1,62 & 0,91 \\
& $\mathrm{~Pb}$ & 1,83 & 0,97 \\
\hline
\end{tabular}


La cantidad de muestra está relacionada con el dispositivo de ultrasonido utilizado. Generalmente, cuando se emplean sondas ultrasónicas se utiliza el peso de muestra de hasta $0,2 \mathrm{~g}$, mientras que para baños de ultrasonidos, este peso puede ser mayor, 0,5 g. Figueiras et al. (2001) y Krishna y Arunachalam (2004) notaron una disminución significativa en la recuperación de algunos metales, al emplear cantidades de muestra mayores a 0,1 a 0,15g, utilizando una sonda ultrasónica. Adicionalmente, Manutsewee et al. (2007) reportaron que se necesita una concentración mayor de ácido para extraer Zn de peces de mejillón y para extraer $\mathrm{Zn}, \mathrm{Cd}$ y $\mathrm{Cu}$ de pescado.

Además del tamaño de muestra y la concentración de los ácidos empleados, la temperatura, es otro factor importante en la digestión de muestras por ultrasonido; tal como se observa en el trabajo de Arain et al. (2007), en los cuales se reporta que la temperatura del baño de agua ultrasónico influyó en la recuperación de $\mathrm{Pb}$ en muestras de peces. Temperaturas de alrededor de $50^{\circ} \mathrm{C}$ normalmente aumentan la eficacia de extracción, pero cuando la temperatura del medio de extracción es similar a la temperatura de bullición de la fase líquida, la eficiencia de sonicación disminuye debido al descenso de la tensión superficial del medio y aumento de la presión de vapor dentro de las burbujas, lo cual causa una reducción de las ondas de choque (Elik, 2005).

\section{CONCLUSIONES}

El método de digestión y extracción de metales pesados, asistido por ultrasonido es viable y recomendable para determinación de $\mathrm{Pb}$ y $\mathrm{Cd}$ en pastos, ya que la recuperación es superior al 97\%.

La determinación de $\mathrm{Cd}$ y $\mathrm{Pb}$ por el método de ultrasonido y cenizas es muy similar, pero se recomienda el método de ultrasonido, ya que es ambientalmente amigable, requiriendo menos tiempo de análisis, así como menor cantidad de solventes para la determinación de los metales y menor consumo de energía.

\section{AGRADECIMIENTOS}

Los autores agradecen al Departamento Administrativo de Ciencias y Tecnología (COLCIENCIAS), de la Republica de Colombia, por su apoyo financiero a esta investigación bajo el macroproyecto titulado "Determinación de residuos de metales pesados en suelo, pastos, agua y tejidos de bovinos expuestos en áreas de alto grado de contaminación y detección de puntos críticos para su control" y al Laboratorio de Control de Calidad de la Universidad de Pamplona, por su apoyo en la ejecución de este trabajo.

\section{REFERENCIAS BIBLIOGRÁFICAS}

ALEIXO, P.C.; SANTOS, J. D.; TOMAZELLI, A.C.; RUFFINI, I.A.; BERNDT, H. D; RUG, F.J. 2004. Cadmium and lead determination in foods by beam injection flame furnace atomic absorption spectrometry after ultrasoundassisted sample preparation. Anal. Chim. Acta. 512:329 - 337. doi: org/10.1016/j.aca.2004.02.049

ARAIN, M.B.; KAZI ,T.G.; JAMALI, M.K.; JALBANI, N.; AFRIDI, H.I.; SARFRAZ, R.A.; SHAH, A.Q. 2007. Determination of toxic elements in muscle tissues of five fish species using ultrasound-assisted pseudodigestion by electrothermal atomic absorption spectrophotometry: optimization study. Spectrosc. Lett. 40:861 - 878. doi: 10.1080/00387010701521868.

BAKKALI, K.; RAMOS, M. N.; SOUHAIL, B.; BALLESTEROS, E. 2009. Characterization of trace metals in vegetables by graphite furnace atomic absorption spectrometry after closed vessel microwave digestion. Food Chem. 116:590 - 594. doi: org/10.1016/j.foodchem.2009.03.010

BONANNO, G; BORG, J.A; DI MARTINO, V. 2017. Levels of heavy metals in wetland and marine vascular plants and their biomonitoring potential: A comparative assessment. Sc. of The Total Env. 576:796-806. doi: 10.1016/j.scitotenv.2016.10.171

BRUNORI, C.; IPOLYI, I.; MACALUSO, L.; MORABITO R. 2004. Evaluation of an ultrasonic digestion procedure for total metal determination in sediment reference materials. Anal. Chim. Acta. 510:101 - 107. doi. org/10.1016/j.aca.2003.12.049 
CESPÓN, R.; YEBRA, B, Y. 2008. Determination of trace metals in urine with an on-line ultrasound-assisted digestion system combined with a flow-injection preconcentration manifold coupled to flame atomic absorption spectrometry. Anal. Chim. Acta. 609:184 - 191. doi: 10.1016/j.aca.2008.01.002

COBB, G.; SANDS, K.; WATERS, M.; WIXSON, M.; DORWARD, K, E. 2000. Accumulation of heavy metals by vegetables grown in mine wastes. Environ. Toxicol. Chem. 19:600 - 607. doi: 10.1002/etc.5620190311

DUONG, T.; BYEONG, K. L. 2011. Determining contamination level of heavy metals in road dust from busy traffic areas with different characteristics. J. Environ. Manage. 92:554 - 562. doi: org/10.1016/j.jenvman.2010.09.010

ELIK, A. 2005. Ultrasound assisted pseudo-digestion of street dust samples prior to determination by atomic absorption spectrometry. Talanta. 66:882 - 888. doi: org/10.1016/j.talanta.2004.12.050

FERRÉ, H. N.; SCHUHMACHER, M.; LLOBET, J.; DOMING0, J. 2007. Metales pesados y salud. Mapfreguridad. 108:50 - 58.

FIGUEIRAS, A.V.; LAVILLA, I.; BENDICHO C. 2001. Ultrasound-assisted solubilization of trace and minor metals from plant tissue using ethylenediaminetetraacetic acid in alkaline medium. Fresenius J. Anal. Chem. 369:451 - 456. doi: 10.1007/s002160000648

JAMALI, M.K.; KAZI, T.G.; ARAIN, M.B.; AFRIDI, H.I.; JALBANI, N.; MEMON, A. 2007. Heavy metal contents of vegetables grown in soil, irrigated with mixtures of wastewater and sewage sludge in pakistan, using ultrasonic-assisted pseudo-digestion. J. Agron. Crop Sci. 193:218 - 228. doi:10.1111/j.1439-037X.2007.00261.x

KABATA, P. A. 2011. Trace elements in soils and plants. 4a. ed. CRC Press, Boca Raton, FL. 505p.

KANTHALE P, M.; GOGATE, PR.; PANDIT, A.B.; WILHELM, A.M. 2003. Mapping of an ultrasonic horn: link primary and secondary effects of ultrasound. Ultrason. Sonochem. 10:331 - 335. doi: 10.1016/S13504177(03)00104-4
KAZI, T.; JAMALI, M.; ARAIN, M; AFRIDI, H.; JALBANI, N.; SARFRAZ, R.; ANSARI R. 2009. Evaluation of an ultrasonic acid digestion procedure for total heavy metals determination in environmental and biological samples. J. Hazard. Mater. 161: 1391 - 1398. doi: 10.1016/j. jhazmat.2008.04.103.

KRISHNA, M.V.B.; ARUNACHALAM, J. 2004. Ultrasoundassisted extraction procedure for the fast estimation of major, minor and trace elements in lichen and mussel samples by ICP-MS and ICP-AES. Anal. Chim. Acta. 522:179 - 187. doi: org/10.1016/j.aca.2004.07.006

MANUTSEWEE, N.; AEUNGMAITREPIROM, W.; VARANUSUPAKUL, P.; IMYIM A.F. 2007. Determination of $\mathrm{Cd}, \mathrm{Cu}$, and $\mathrm{Zn}$ in fish and mussel by AAS after ultrasound-assisted acid leaching extraction. Food Chem. 101:817- 824. doi: 10.1016/j.foodchem.2005.12.033.

MEREY, R.; MASRI, M.; BOZOU, R. 2002. Cold ultrasonic acid extraction of copper, lead and zinc from soil samples. Anal. Chim. Acta, 452:143 - 148. doi:10.1016/ S0003-2670(01)01431-3

MILLER, J.; MILLER, J. 2000. Estadística y quimiometría para química analítica. Cuarta Edición. Prentice Hall, Madrid. $271 \mathrm{p}$.

MINKINA, T.; MANDZHIEVA, S.; CHAPLYGIN, V.; BAUER, T.; BURACHEVSKAYA, M.; NEVIDOMSKAYA, D.; SUSHKOVA, S.; ZAMULINA, I. 2016. Content and distribution of heavy metals in herbaceous plants under the effect of industrial aerosol emissions. Journal of Geochemical Exploration. doi: dx.doi.org/10.1016/j. gexplo.2016.05.011.

MIRANDA, D.; CARRANZA, C., ROJAS, C.; JEREZ, C.; FISCHER, G.; ZURITA, J. 2008. Acumulación de metales pesados en suelo y plantas de cuatro cultivos hortícolas, regados con agua del río Bogotá. Rev. Colomb. Cienc. Hortic. 2:180 - 19. doi: http://dx.doi.org/10.17584/ rcch.2008v2i2.1186

MOLLON, L.C; NORTON, G.J; TRAKAL, L; MORENOJIMENEZ, E; ELOUALI, F.Z; HOUGH, R.L; BEESLEY, L. 2016. Mobility and toxicity of heavy metal(loid)s arising from contaminated wood ash application to a pasture grassland soil. Env Pollution. 218:419-427. doi: 10.1016/j.envpol.2016.07.021 
NASCENTES, C.; KORN, M.; ARRUDA, M. 2001. A fast ultrasound-assisted extraction of $\mathrm{Ca}, \mathrm{Mg}, \mathrm{Mn}$ and $\mathrm{Zn}$ from vegetables. Microchem. J. 69:37 - 43. doi: http://dx.doi. org/10.1016/S0026-265X(00)00192-2

NAVARRO, J.; AGUILAR, I.; LÓPEZ, M. J. 2007. Aspectos bioquímicos y genéticos de la tolerancia y acumulación de metales pesados en plantas. Ecosistemas. 16(2):10 - 25.

NEGRONI, M. 2009. Microbiología Estomatológica. Fundamentos y guía práctica. Segunda Edición. Editorial Médica Panamericana, México D.C. 656 p.

PRIEGO, C. F.; LUQUE DE CASTRO, M. 2007A. Ultrasoundassisted digestion: A useful alternative in sample preparation. J. Biochem. Biophys. Methods. 70:299 - 310. doi: http://dx.doi.org/10.1016/j.jbbm.2006.09.006

PRIEGO, C. F.; LUQUE DE CASTRO, M. 2007B. Ultrasound in analytical chemistry. Anal. Bioanal. Chem. 387:249 257. doi: $10.1007 / \mathrm{s} 00216-006-0966-4$

PRIETO, J.; GONZÁLEZ, C.; ROMÁN, A.; PRIETO, F. 2009. Contaminación y fitotoxicidad en plantas por metales pesados provenientes de suelos y agua. Tropical and Subtropical Agroecosystems, 10:29 - 44.

RASCIO, N.; NAVARI, I. F. 2011. Heavy metal hyperaccumulating plants: How and why do they do it? And what makes them so interesting?. Plant Sci. 180:169 - 181. doi: 10.1016/j.plantsci.2010.08.016

RUIZ, J.; LUQUE, J.L.y LUQUE DE CASTRO, M.D. 2003. Dynamic ultrasound-assisted extraction of cadmium and lead from plants prior to electrothermal atomic absorption spectrometry. Anal. Chim. Acta. 480:231 - 237. doi: http://dx.doi.org/10.1016/S0003-2670(02)01658-6

SOYLAK, M.; TUZEN, M.; SANTOS, A.; ANDRADE, M.G.; COSTA, S.L. 2007. Optimization of microwave assisted digestion procedure for the determination of zinc, copper and nickel in tea samples employing flame atomic absorption spectrometry. J. Hazard. Mater. 149:264 - 268. doi: http://dx.doi.org/10.1016/j.jhazmat.2007.03.072

SKOOG, D.; WEST, D.; HOLLER, F.; CROUCH, S. 2005. Fundamentos de Química Analítica. Octava Edición. Thomson, México D.C. 1065 p.
TCHOUNWOU, PB; YEDJOU, CG; PATLOLLA, A.K; SUTTON, DJ. 2012. Heavy metal toxicity and the environment. Molecular, clinical and environmental toxicology. 3: 133-164. doi: 10.1007/978-3-7643-8340-4_6.

TÓTH, G; HERMANN, T.; DA SILVA, M.R.; MONTANARELLA, L. 2016. Heavy metals in agricultural soils of the European Union with implications for food safety. Environ. Int. 88:299 - 309. doi: http://dx.doi. org/10.1016/j.envint.2015.12.017

WANG, X; CHEN, J; YAN, X; ZHANG, J; HUANG, J, ZHAO, J. 2015 Heavy metal chemical extraction from industrial and municipal mixed sludge by ultrasound-assisted citric acid. J. of Industrial and Engineering Chemistry. 27:368 - 372. doi: 10.1016/j.jiec.2015.01.016 